\title{
INMUNIZAR CONTAGIANDO. LA PRÁCTICA DE LA INOCULACIÓN COMO TRATAMIENTO PREVENTIVO FRENTE A LA VIRUELA EN LA CAPITANÍA GENERAL DE CHILE A FINES DEL SIGLO XVIII ${ }^{1}$
}

\author{
Paula Caffarena Barcenilla \\ Universidad Finis Terrae, Facultad de Comunicaciones y Humanidades, Escuela de Historia, Santiago, Chile. \\ pcaffarenab@uft.edu
}

Recibido: 23 noviembre 2015; Aceptado: 11 julio 2016.

Cómo citar este artículo/Citation: Caffarena Barcenilla, Paula (2016), "Inmunizar contagiando. La práctica de la inoculación como tratamiento preventivo frente a la viruela en la Capitanía General de Chile a fines del siglo XVIII", Asclepio 68 (2): p151. doi: http:// dx.doi.org/10.3989/asclepio.2016.23

RESUMEN: Este artículo examina el estado en que se encontraba la inoculación a fines del siglo XVIII en la Capitanía General de Chile, a través de una epidemia de viruela que afectó a Concepción y sus alrededores entre 1789 y 1791 . Una de las preguntas centrales de este estudio es en qué medida la inoculación puede considerarse como el primer tratamiento preventivo para enfrentar la viruela y el papel que la población jugó en ello. Buscamos también determinar la importancia que tuvo la variolización en la configuración y aceptación de la noción de inmunización en la sociedad del periodo, pues más allá del número de personas que se sometieron a la práctica, su implementación se tradujo en la discusión temprana de nociones como prevención y universalidad. Dichas nociones fueron fundamentales en el desarrollo de las posteriores medidas que se utilizaron para enfrentar la viruela en la sociedad chilena.

PALABRAS CLAVES: Viruela; Inoculación; Epidemia; Prevención; Inmunización.

\section{TO IMMUNIZE WHILE INFECTING. THE PRACTICE OF VARIOLATION AS PREVENTIVE TREATMENT AGAINST SMALLPOX IN THE GENERAL CAPTAINCY OF CHILE IN THE LATE EIGHTEENTH CENTURY}

ABSTRACT: This article delves onto the state of variolation at the end of the eighteenth century in the General Captaincy of Chile, by studying a smallpox epidemic which affected Concepción and its surrounding areas. One central question of this study is to what extent variolation might be regarded as the first preventive treatment against smallpox and the role played by the population. It also aims to determine the importance of variolation for both the configuration and acceptance of the notion of immunization by the society of the period. This is because beyond the amount of persons who adopted this practice, its implementation led to an early debate on notions such as prevention and universality. Such notions were fundamental for the development of further measures against smallpox in Chilean society.

KEYWORDS: Smallpox; Variolation; Epidemic; Prevention; Immunization. 


\section{INTRODUCCIÓN}

El estudio de la inoculación o variolización en los territorios hispanoamericanos representa un ámbito de investigación en gran medida inexplorado, pues a pesar de que los primeros registros de esta práctica datan de 1760, poco sabemos respecto a su impacto en la lucha contra la viruela y sobre su real alcance en la población del periodo. La escasez de fuentes, posiblemente sea la mayor dificultad para trabajar estos temas, pues no contamos con datos estadísticos ni informes continuos respecto a los progresos de la inoculación. Este trabajo busca ser un aporte en este sentido, analizando el caso la Capitanía General de Chile y específicamente la epidemia que afectó a Concepción y sus alrededores entre 1789 y 1791 . Esto nos permitirá examinar una situación concreta en la cual la variolización se puso en práctica y fue recomendada por las autoridades. Una de las preguntas centrales de este estudio es en qué medida la inoculación puede considerarse como el primer tratamiento preventivo para enfrentar la viruela y el papel que la población jugó en ello. Del mismo modo, nos interesa determinar si la implementación de la inoculación en la Capitanía General de Chile dio origen a aplicaciones permanentes o esporádicas y cómo éstas pudieron influir en el desarrollo y aceptación de la noción de inmunización en la sociedad del periodo, pues más allá del número de personas que se sometieron a la práctica, su implementación se tradujo en la discusión temprana de nociones como prevención y universalidad.

\section{REFERENTES EN LA INOCULACIÓN DE LA VIRUELA: MIEDOS, CONTROVERSIAS Y MASIFICACIÓN}

El siglo XVIII no solo marcó cambios a raíz del interés de las autoridades en la salud de la población, sino también por la masificación de nuevas técnicas que permitieron combatir la viruela ${ }^{2}$. Una de ellas fue la variolización, que bajo el principio de la inmunización consiguió precaver de viruela a quienes se sometieron a dicho tratamiento. En palabras del médico peruano Cosme Bueno, el fin de la inoculación era provocar en el paciente "unas viruelas benignas, de cuyas consecuencias no se sigan los peligros y accidentes, que suelen experimentarse en las viruelas naturales, eligiendo para ello las circunstancias del tiempo, y otras, que son más al propósito para su buen éxito ${ }^{3}$ (Cos- $^{-}$ me Bueno, 1777, p.7). Esta técnica era practicada en China e India desde el año 2550 A.C. y hacia 1721 se introdujo en Europa cuando la esposa del embajador inglés en Constantinopla, Lady Mary Worthley Montague, la llevó a Inglaterra después de haber hecho inocular a su hijo por una mujer turca (Piedrola, 1977, p.354). Los médicos Emmanuel Timoni y Hans Sloane vieron sus beneficios y la difundieron en dicho lugar.

Casi de modo paralelo, hay registros de ella en América del Norte, específicamente en la ciudad de Boston, cuando el reverendo Cotton Marther puso en práctica la inoculación para combatir una epidemia que había sido introducida por un barco de esclavos africanos. De acuerdo al estudio realizado por Sheldon Watts, Cotton aprendió la técnica alrededor de quince años antes, cuando adquirió un esclavo africano que «temeroso de que éste estuviera enfermo, le preguntó si alguna vez había tenido viruelas. El esclavo le respondió que sí y que no, que como todos los de su edad, lo habían inoculado cuando niño y había tenido entonces un brote benigno que le dio inmunidad vitalicia» ${ }^{4}$ (Watts, 1977, p. 113). Opinión distinta tienen Pilar León y Dolores Barretino, quienes señalan que Cotton Marther y el médico Zabdiel Boyslton «tenían noticias de la práctica por la lectura de publicaciones inglesas que trataban sobre las experiencias de Timoni y de otros médicos» (León, 2007, p.212). Otras fuentes del periodo, como por ejemplo los escritos de Charles Marie de la Condamine, también dieron cuenta de que los médicos de Boston habían conocido la variolización antes o de modo paralelo que Inglaterra, de tal manera que es posible aceptar la tesis también planteada por Peter Skold respecto a que «la inoculación había sido practicada en muchas partes fuera de Europa» (Skold, 1996, p. 249).

Estos antecedentes cuestionan la noción tradicional respecto a que la inoculación fue difundida desde Inglaterra al resto del mundo occidental, poniendo en duda la idea de una transferencia unidireccional del conocimiento médico. Tanto los contactos comerciales como las conexiones sociales y culturales que había en aquel periodo, permiten pensar que la masificación de la variolización no se explica por la idea de un descubrimiento. Más bien, se trató probablemente de focos simultáneos, que no siguieron un recorrido único, y que se explican por las interconexiones que existieron durante el siglo XVIII, que se tradujeron en un mayor dinamismo en la adquisición y práctica de ésta.

Hasta la masificación de la vacuna a partir de 1796, tanto la inoculación como todos los otros tratamientos contra la viruela, funcionaron de modo paralelo ${ }^{5}$. Sin embargo, la variolización abrió un debate práctico y teórico sobre los límites del saber y la práctica médica. De acuerdo al médico español Francisco Gil, inocular se trataba de "comunicar la materia variolosa de unos en otros» (Gil, 1784, p.30), de modo que 
preguntas como si acaso era legítimo prevenir la viruela introduciendo la misma enfermedad en el ser humano predominaron en el debate. Esta idea, que hoy conocemos bajo la noción de «inmunización», no fue aceptada con facilidad por la sociedad del periodo. De acuerdo a Emma Spary, el principal problema es que al fundamentarse la variolización en dicho principio, se contrapuso al modelo humoral que explicaba la salud y la enfermedad en función del restablecimiento del equilibrio de los humores (Spary, 2011, p.93). La inoculación, entonces, representó una nueva forma de pensar la enfermedad y también de cómo enfrentarse a ella, pues ya no se trataba de curar a quien padecía viruela, sino de evitar que la población se infectara.

Si bien la medida generó interés dado que era el único medio que aseguraba proteger al ser humano de la viruela, su uso no fue aceptado por todos dados los riesgos que implicaba variolizar. En España, por ejemplo, las controversias respecto a esta técnica se expresaron en la publicación de una serie de tratados médicos a favor y en contra de la inoculación. Quienes se oponían a ella argumentaron que la técnica no garantizaba el no padecer viruela y que, por el contrario, la persona padecería una viruela de carácter suave, que si bien le permitiría conseguir la inmunización, podía dejar secuelas que la acompañarían de por vida. Asimismo, la inoculación no evitaba el contagio, dado que la persona inoculada podía propagar en otros la enfermedad, al igual que ocurría con la viruela natural. También hubo cuestionamientos de índole religioso, como por ejemplo, si era lícito o no introducir viruela de manera artificial ${ }^{6}$. Fue el científico francés Charles Marie de la Condamine quien mejor argumentó en su favor, señalando que de acuerdo a la teología cristiana, la vida se consideraba como un depósito de conservación del cual estábamos obligados a cuidar, por tanto, «si ese depósito corre riesgo de sernos quitado, es nuestro deber, por todos los medios que la prudencia puede sugerir, ponerlo al abrigo de la invasión» (De La Condamine, 1783 , p. 192). Bajo este razonamiento, la inoculación no contravenía la ley de Dios, lo que permitió a médicos como Cosme Bueno defender su utilización en el marco de la monarquía hispana.

\section{DIFUSIÓN, TEMOR Y RESISTENCIA. LA INOCULACIÓN EN LA CAPITANÍA GENERAL DE CHILE}

En Hispanoamérica la inoculación fue también practicada y aceptada por los médicos en la segunda mitad del siglo XVIII. Cosme Bueno señaló que la primera variolización se realizó en Popayán hacia
1738, mientras que Paula De Demerson ha mostrado que ésta se puso en uso desde al menos 1746 . Al respecto, señala además que «en una carta dirigida al Semanario de Agricultura y Artes José Celestino Mutis, residente en Santa Fe de Bogotá, confirma que tan importantísima práctica se ha hecho general en el nuevo Reino de Granada» (De Demerson, 1993, p.30). En Caracas la inoculación contra la viruela fue introducida por el doctor Juan Perdomo en 1765 (Amodio, 1997, p. 115), mientras que en el Río de la Plata la primera referencia a la inoculación es de 1796 cuando Miguel Gorman comenzó a practicarla. Para el caso de la Capitanía General de Chile su uso se inició en 1765 por Fray Pedro Manuel Chaparro de la Orden San Juan de Dios, mientras que en el Virreinato del Perú, fue Fray Domingo de Soria, también de la Orden San Juan de Dios, el primero en practicar la variolización en Lima en $1766^{7}$.

Tanto la bibliografía como los documentos del periodo estudiado muestran que las primeras inoculaciones que se realizaron en Chile datan de 1765 y que fue Pedro Manuel Chaparro quien las llevó a cabo. El cronista Carvallo Goyeneche señaló que fue este sacerdote quien «comenzó la inoculación con tanto acierto que fue el iris que serenó aquella horrible tempestad. Excedieron el número de cinco mil las personas inoculadas y ninguna pereció» (Carvallo y Goyeneche, 1875, p. 311). Además de estas informaciones, en una exposición que Chaparro realizó para que se le nombrara protomédico, argumentó su idoneidad para el cargo aludiendo que había sido él quien introdujo la inoculación ${ }^{8}$.

No sabemos con certeza cómo Chaparro aprendió la técnica. Su figura se ha asociado a la de un autodidacta que pudo haber adquirido la técnica en base a sus lecturas, lo cual lo vincula con la circulación por Hispanoamérica de diversos escritos que pudieron estar a su alcance (Gutiérrez, 2008, p.29). Esto mismo lo recordó Chaparro años después en una comunicación a la Universidad de San Felipe fechada en 1776, donde enfatizó que «inquiriendo mi desvelo y estudio algún modo fácil y de más acertado éxito para la curación de viruelas puse por obra el experimento de la inoculación (...) cuyos éxitos y resultados puede decir el público» (Gutiérrez, 2008, p.30).

Sobre las cifras de inoculados que comúnmente se mencionan en la bibliografía, es difícil saber qué tan confiables son, pues solo disponemos de unos pocos documentos que se refieren directamente al número de personas que se sometieron a la variolización. Una fuente importante al respecto es un oficio de la 
Real Audiencia, donde se expresó la confianza en la técnica. Se afirmó, también, que durante siete años Chaparro inoculó a cerca de diez mil personas, de las cuales solo habían muerto cuatro y, casi con certeza, por motivos ajenos a la inoculación ${ }^{9}$.

Todo ello muestra que a pesar de que la variolización no contó con un registro detallado ni fue parte de una iniciativa coordinada por la autoridad central, se practicó en la Capitanía General de Chile y dependió de la voluntad de quienes quisiesen someterse al tratamiento preventivo. Gracias a algunos testimonios disponibles, podemos comprender el alcance que ésta tuvo en la Capitanía General de Chile. En 1796, Manuel de Salas se refirió a las ventajas de Chile en un escrito dirigido al Ministro Diego Gardoqui, caracterizando al territorio como un lugar «en que jamás truena ni graniza, con unas estaciones regladas que rarísima vez se alteran, sembrado de minas de todos los metales conocidos, con salinas abundantes, regados de muchos arroyos, manantiales y ríos, que a cortas distancias desciendes de la cordillera..." (De Salas, 1910, p. 45). Salas presentó a Chile como un país ideal, donde incluso planteó que «se han olvidado los estragos de la viruela por medio de la inoculación» (De Salas, 1910, p. 45), pues había disminuido la mortalidad causada por esta enfermedad. Sobre esto último, no podemos estar tan seguros de que así haya sido, dado que precisamente dos años después de estas palabras una epidemia en Concepción cobró más de mil vidas. Por otra parte, no tenemos evidencias que demuestren que en Chile la inoculación abarcó a un gran número de personas ni que se hubiese puesto en práctica algún programa de inoculación masiva. Al respecto, Diego Barros Arana ha planteado que si bien fue una medida preventiva útil, fue aceptada con muchas resistencias y que se difundió mayormente entre las clases acomodadas de las ciudades con mayor cantidad de habitantes. La situación era distinta tanto en los campos como en los pueblos más pequeños, pues en aquellos lugares era difícil aplicar la inoculación ya fuese por falta de medios o de personas que supieran hacerlo. Además, «las gentes se resistían tenazmente a someterse a ella, persuadidas de su ineficacia, o de que era a veces origen de gravísimas enfermedades» (Barros Arana, 2002, p. 270).

Esta resistencia estuvo estrechamente vinculada a la incertidumbre que generaban los nuevos tratamientos, así como al desconocimiento de los efectos de inocular y al temor al contagio, lo cual quedó de manifiesto en otras ocasiones. Por ejemplo, en una sesión del cabildo de octubre de 1789 , se solicitó a Am- brosio O'Higgins que publicara un bando por el que se prohibiera inocular durante las fiestas reales que se realizarían en honor a Carlos IV, destacando que no era conveniente que se llevara a cabo esta práctica, pues muchas personas vendrían a la capital «entre las cuales serán raras las personas que hayan tenido las viruelas, recelándose justamente que de continuarse la inoculación se propague dicha enfermedad con peligro de las personas que no la han experimentado ${ }^{10}$. Esta petición del cabildo muestra que se temía que por medio de la inoculación la viruela se propagara, de modo que O'Higgins emitió el decreto solicitado, prohibiendo la inoculación de viruelas durante las fiestas reales, "siendo de la obligación del protomédico el cumplimiento de esta orden ${ }^{11}$.

El temor al contagio fue un factor determinante en la resistencia que generó variolizar, pues, como veremos en el caso de la epidemia de 1789 , no fueron argumentos religiosos o de valor, como generalmente se piensa, los que se esgrimieron para rechazarla, sino que aquéllos vinculados a los riesgos propios de esta práctica.

\section{UNA PRERROGATIVA DE LOS MÉDICOS. LA INOCULA- CIÓN DURANTE LA EPIDEMIA DE 1789 EN CONCEPCIÓN}

Durante la epidemia que azotó a Concepción en 1789, la inoculación fue un medio recomendado para enfrentarla, tanto por el gobernador Ambrosio O'Higgins como por el Protomedicato, pues ambos asumieron que era el medio más efectivo de prevenir el contagio. O'Higgins autorizó la inoculación, dado que al recibir las noticias de Francisco de la Mata Linares tomó la decisión de enviar al inoculador Juan José Morales a la zona afectada. Morales no era médico sino un práctico de medicina, que en palabras de O'Higgins, «en esta capital ejercita con acierto la inoculación y curación de las viruelas» ${ }^{12}$. Morales tenía experiencia en la lucha contra la viruela, pues durante la epidemia de 1787 que afectó a Santiago, fue encargado de atender a los enfermos del hospital provisional instalado en la casa de huérfanos. Si bien en los documentos no se especificó quienes debían ser inoculados, es posible deducir que todos quienes la quisieran podían obtenerla, ya que no se estipularon costos ni condiciones especiales para el inoculado, salvo las vinculadas al procedimiento ${ }^{13}$.

La puesta en práctica de la inoculación en la epidemia de 1789 requiere estudiarse con atención, dado que no se trató de un programa obligatorio de inoculación, si no de poner a disposición la medida para quienes quisieran utilizarla. No obstante, Ambrosio O’Higgins reconoció que había médicos que se opo- 
nían a ella, de modo que señaló que su ejecución «se premedite con la seriedad que exigen el caso, y sus incidencias, a presencia del ánimo de los mismos pacientes, y de todas las demás circunstancias que observara ${ }^{14}$. Igualmente, cuando el Protomedicato recomendó la variolización para enfrentar dicha epidemia, lo hizo bajo las mismas directrices planteadas por Francisco Gil. De este modo, la primera indicación que dieron los protomédicos fue la de instalar dos hospitales provisionales en función de la dirección del viento. Por ello, el hospital de preparación debía situarse a barlovento de la ciudad de modo que «los individuos que se dispongan a la operación, no se contagien en esta estación por las exhalaciones de la ciudad", mientras que el otro, donde las personas se inoculaban, debía situarse a sotavento, "para que los inoculados, no añadan miasmas al pueblo» ${ }^{15}$.

Los hospitales de inoculación tuvieron por objeto reducir la posibilidad que el inoculado contagiara a otros la enfermedad, así como no continuar infectando el aire de la ciudad. Es por ello que el Protomedicato recomendó instalar estos hospitales en la Isla de la Quiriquina, lugar que tanto en 1759 como en 1760 fue usado para que la fragata Soplo de Lero y el navío Begonia, respectivamente, realizaran la cuarentena por arribar a puerto con enfermos de viruela. Una vez establecidos estos hospitales, los protomédicos precisaron la importancia de que tanto los inoculados como los infectados por viruela natural estuvieran bajo el "cuidado de un médico de profesión, porque una y otra mal manejadas sacrificarán muchas vidas» ${ }^{16}$. La importancia de que los médicos estuvieran a cargo tanto de los inoculados como de los enfermos de viruela natural ya había sido expresada en un acuerdo del Cabildo de Santiago del 9 de octubre de 1789, en el que se advirtió que el procedimiento de inocular debía hacerse «por personas peritas que siguieran las «observancias prevenidas para ellas y generalmente practicadas en la Europa, disminuyéndose con ellas los estragos padecidos antes de haberse puesto en práctica la referida inoculación ${ }^{17}$.

La precisión respecto de que solo las personas peritas podían llevar a cabo la inoculación formó parte del esfuerzo por regular el ejercicio de la medicina propio del siglo XVIII. En el caso de dicha práctica, se estableció, por ejemplo, que solo la podían realizar médicos o prácticos de medicina que supieran aplicar la técnica, como Juan José Morales y Pedro Manuel Chaparro. El primero de ellos, si bien no era médico, había recibido licencia del cabildo para curar enfermos, mientras que el segundo obtuvo el grado de doctor en la Universidad de San Felipe en 1772. Esta disposición fue importante, pues fue la primera vez, al menos durante esta epidemia, que se estableció que el cuidado de los enfermos debía quedar a cargo de un médico. Esto no es extraño si pensamos que precisamente entre las funciones del Protomedicato estuvo el interés por disciplinar el ejercicio de la medicina y la inoculación era, además, parte de los tratamientos médicos usados contra la viruela. Se practicaba en Chile hace veinticuatro años y al parecer había adquirido cierta popularidad, pues, a pesar de los riesgos, hubo personas que inocularon y otras que se sometieron a dicho tratamiento.

Por otro lado, las tensiones frente a la inoculación salieron también a la luz a raíz de esta epidemia, ya que aunque O'Higgins y el Protomedicato recomendaron la medida, el Fiscal de la Real Audiencia, Joaquín Pérez de Uriondo se opuso enérgicamente al uso de ella en Concepción ${ }^{18}$. Los argumentos que empleó plantearon que inocular podía agravar la situación de la ciudad por la posibilidad de contagio que implicaba. De este modo y haciendo referencia a la epidemia que había afectado a Santiago en 1787, explicó que en dicha oportunidad la viruela había llegado a Santiago desde la ciudad de Mendoza por dos peones de arriero que exhibían unas pústulas de viruela de buena calidad. Frente a ello, muchos vecinos usaron el remedio precautorio de la inoculación, y por este medio lo que al principio era una pequeña chispa que en su origen pudo muy bien sofocarse del todo, se hizo rápidamente un incendio tan grande que en pocos meses se comunicó a innumerables vivientes ${ }^{19}$.

Esta situación de alerta que expuso el fiscal, enfatizó los riesgos de la inoculación en tanto puso en evidencia la dificultad de los inoculadores para identificar las pústulas de viruela benigna y extraer de allí el pus para inocular. De acuerdo a su testimonio, el gran riesgo se encontraba en dicha operación, pues si se escogía el pus incorrecto se corría el riesgo de infectar a un gran número de personas de viruela maligna. Si bien el fiscal reconoció que la técnica cumplía con el objetivo de inmunizar contra la viruela, pues «libra ciertamente del riesgo de padecer de nuevo viruelas, y que es contingencia sumamente rara el que alguno muera de las artificiales o ingestadas ${ }^{20}$, manifestó que la situación en la que se encontraba Concepción no era la más adecuada para implementarla, pues la ciudad se encontraba en medio de un brote epidémico «en la cual han muerto muchos de los mismos que procuraban precaverlas con la inoculación, y han contagiado a infinitos, que tal vez no las hubieran padecido sino se hubiese dado lugar a la propagación difundida violen- 
tamente con la inoculación ${ }^{21}$. Así, más que rechazar la inoculación en tanto remedio preventivo, el fiscal destacó que la situación particular de Concepción no era la apropiada para aplicarlo. Con la experiencia conocida, el fiscal recomendó no poner en práctica la variolización ante la epidemia de Concepción, pues señaló que "... de lo que ha enseñado al fiscal la experiencia en esta ciudad, nunca será de opinión que en aquella se usase el remedio precautorio de la inoculación porque juzga que ese no es el modo de extinguir la epidemia ${ }^{22}$. El temor de que la inoculación aumentara el contagio fue un factor relevante en la puesta en marcha de la inoculación. Finalmente, sin embargo, bajo recomendación del Protomedicato y a pesar de dichas objeciones, la variolización se practicó en Concepción a partir de octubre de 1789 , cuando aún la epidemia se encontraba en su fase inicial.

Las razones que permitieron que esta técnica se impusiera y venciera los temores que el propio fiscal de la Real Audiencia manifestaba, podemos encontrarlas en las palabras del Intendente y en las discusiones de las sesiones del cabildo realizadas en la ciudad, dado que hacia octubre de 1789 aun había una gran cantidad de casos de viruela benigna en Concepción. Es difícil saber a ciencia cierta si la inoculación influyó o no en el agravamiento de la epidemia y si existió una correlación entre el aumento de los contagiados y la inoculación. Sin embargo, los documentos muestran que la variolización se practicó siguiendo las instrucciones de Francisco Gil, lo que se tradujo en el establecimiento de hospitales fuera de la ciudad. Según un informe del cabildo de octubre de 1789, la inoculación hacía «felices progresos, sin que se deba entender esta exposición en ningún modo, influyente o directiva a los ánimos, sino especialmente voluntaria» ${ }^{23}$. A la luz de los antecedentes, es poco probable que la inoculación haya tenido una repercusión directa en el aumento de los contagiados, dado que no fue una práctica masiva y es probable que el contagio natural haya bastado para agravar la epidemia. Por otra parte, aunque la actitud de la población frente a la inoculación es difícil de rastrear, sabemos que algunos accedieron a ella cuando la posibilidad de contagio era inminente, mientras que otros se resistieron. Francisco de la Mata Linares dio cuenta de esta situación señalando que, aunque la inoculación era voluntaria "y su importancia muy grande para cada particular, de aquí nace, que casi todos los que se han inoculado, han dilatado la operación hasta verse rodeados de los peligrosos estragos de la viruela natural» ${ }^{24}$. Otro factor importante fue el efecto generado cuan- do circulaba la noticia de que algún habitante de la ciudad con cierta notoriedad social era inoculado, lo que llevaba a otros a imitar su comportamiento:

Doña Isabel de Santa María, y su familia, fueron los primeros que se inocularon, y viendo con la facilidad que se curaron todos empezaron varios a ejecutar lo mismo: creo que a esta señora le es la Concepción y su Obispado, deudores de este beneficio, pues seguramente se han libertado muchos de ser víctimas de la peste, como lo confiesan no con poco dolor algunas familias quienes no padecerían las indigencias a que han quedado expuestas por no haber admitido aquel arbitrio ${ }^{25}$

Los progresos de la inoculación en Concepción también fueron parte del informe que Juan José Morales envió a O'Higgins, el que si bien no incorporó el número de variolizados, sí expresó los términos en que se puso en marcha. Este informe fue escrito un mes después de que esta práctica llegara a la ciudad y señaló que si bien «no faltó parecer en contra, con todo la mayor parte convino en que se inoculase quien quisiese ${ }^{26}$. Además, dio cuenta de que su labor no solo consistió en inocular sino también en que «instruyese yo aquí a los facultativos en el método de inocular para que habido este conocimiento pasase con la mitad de los medicamentos a socorrer la ciudad de Chillán ${ }^{27}$.

De acuerdo a los documentos revisados, la ciudad de Chillán enfrentó una viruela más mortal que la de Concepción, pues en cuarenta días entre julio y agosto habían muerto ciento seis personas ${ }^{28}$. Esto explica que el envío de Morales a la zona tuviera por objeto conformar una red de inoculadores que cubrieran los distintos lugares afectados. De este modo, Morales partió a Chillán con una asignación de treinta pesos mensuales, gratificación que fue entregada por la ciudad de Concepción, por lo que el Intendente señaló que, «aunque corta en realidad para él, podrá ser bastante fuerte para la ciudad, si su existencia en ella fuese larga, como es presumible, ya sea considerando el paso natural de la epidemia o ya el de la inoculación ${ }^{29}$. Este plan no fue del todo exitoso, pues Francisco de la Mata Linares indicó que a pesar de encontrarse en Chillán, Morales no había podido introducirla «porque no ha encontrado viruela bastante benigna para ello». Así, el Intendente recomendó a Morales volver a la ciudad de Concepción para que «después de instruir a las personas que allí asistan a los enfermos, se venga a enseñar y practicar aquí la inoculación mientras todavía se mantenga buena la viruela.... ${ }^{30}$. Morales siguió esta recomendación y continuó inoculando en la ciudad así como llevando a cabo los otros tratamientos que el Protomedicato había recomendado. 
En mayo de 1790, Juan Ubera, capellán del cuerpo de dragones y que a la vez era médico y cirujano, puso nuevamente en debate el tema de la inoculación. Específicamente, se preguntaba si se debía proceder o no a inocular las nuevas zonas amenazadas por la viruela. En el caso del partido de Rere, se mostró proclive a inocular lo antes posible, considerando que más que los resultados negativos fue el temor de la población hacia esta técnica nueva lo que frenó su avance ${ }^{31}$. Ubera se mostró a favor de la inoculación argumentando los buenos resultados que había tenido en Concepción y destacando que a pesar de que "dirán que es temeridad introducir una enfermedad donde no la hay", precisaba que había visto "con bastante dolor, los funestos destrozos que acaba de hacer en la Concepción la viruela natural, por no haber querido sus habitantes tomar el consejo de la inoculación ${ }^{32}$. Su confianza en la variolización se complementó con las recomendaciones de Francisco Gil, pues al igual que la indicación que el Protomedicato envió a Concepción, mostró su preocupación respecto a que la técnica se aplicara con cautela y en los lugares habilitados para ello. En este sentido, señaló que «no es señor mi pensamiento que proceda a la inoculación en la Isla de la Laja, y plazas de la frontera indistintamente, sino que se pongan hospitales en parajes oportunos como llevo dicho para el efecto Yumbel donde hay cuarteles a propósito de ir bien preparados para la inoculación ${ }^{33}$.

La aplicación de la inoculación implicó un nuevo debate y un nuevo pronunciamiento del Protomedicato, que si bien había aceptado su uso en Concepción, en esta oportunidad no era de igual parecer. En esta oportunidad se planteó que la amenaza de epidemia para la zona mencionada apareció en junio, y se debía esperar hasta la primavera para inocular, ya que el invierno no era la estación adecuada para hacerlo ${ }^{34}$. Los protomédicos, a pesar de que confiaban en que la inoculación era el mejor remedio para precaverse de la viruela, recalcaron que «debe hacerse (como todas las cosas), en un tiempo, modo y proporción debida, porque de lo contrario se sacrificarán a los mismos que se procura libertar. El tiempo presente, señor que es el del invierno, es el más contrario a esta operación... ${ }^{35}$. Los reparos del Protomedicato no eran sobre la utilidad de la inoculación, si no sobre el momento apropiado en que se debía aplicar; sin embargo, dado el avance de la enfermedad y ante «el deseo de los jefes del reino por el bien del Estado" tanto Ubera como los protomédicos, «han resuelto proponer el método de la inoculación $\aleph^{36}$.
En esta oportunidad se convocó a una asamblea pública de los jueces, oficiales militares y vecinos de la Plaza de Los Ángeles y de la Isla de Laja que eran las zonas amenazadas por la epidemia, con el objetivo de que acordaran y dieran su parecer «con la libertad y fundamentos sobre el uso de la inoculación ${ }^{37}$. Esta consulta se explica porque poner en práctica la inoculación tenía sus riesgos y aunque algunos decidieran no utilizarla, el contagio podía aumentar. Además, el éxito y seguridad de la práctica dependía del seguimiento estricto del procedimiento. Precisamente, esta fue la razón por la cual la asamblea que se reunió el 4 de julio decidió no poner en práctica la inoculación, pues se puso en duda que quienes acudiesen a inocularse cumpliesen la cuarentena en los hospitales establecidos. La asamblea señaló que si el hospital se pusiera en Yumbel, "haría muy pocos o ningunos, y aun cuando fuesen muchos, no podrían lograrse el fin en dilatado tiempo, y sucedería sin duda que los inoculados incapaces de subsistir el de la cuarentena de la otra banda de la laja se volverían a sus casas, aventurándose al río ${ }^{38}$. La otra alternativa consistía en instalar hospitales en la Isla de la Laja, la que tampoco fue bien acogida, ya que

sería sin duda más prontamente infestada por las dificultades de mantener en la preciosa cuarentena a los inoculados, quienes al momento de verse libres del accidente se mudarían a sus casas por más guardias y precauciones que se tomasen, tanto por su barbarie como por las escaseces que allí tendrían en los cuarenta o cincuenta días de su permanencia, y de este modo, vendría a contagiarse el país y mucho más si se permitía que se inoculasen a discreción en sus estancias, pues en este caso para salvar la Isla de la frontera y extendida población de campaña sería menester muchísimos facultativos llenos de celo y robuste, para correr su dilatada extensión ${ }^{39}$.

Durante 1791 la viruela se mantuvo en la zona de la frontera afectando a la población del lugar. En el estudio realizado por Fernando Casanueva sobre esta fase de la epidemia, no hay referencias a que la inoculación hubiese sido aplicada. Si bien se refiere al testimonio de Ambrosio O'Higgins que mandó tener «preparado cuanto sea posible para ocurrir a tiempo con el remedio de la inoculación precavida en el evento de suceder aquella desgracia que me será muy sensible ${ }^{40}$, no hay evidencias que muestren que efectivamente se haya inoculado en aquella zona. Por una parte, como señala Casanueva, porque los indígenas siempre estaban «recelosos de todo de lo que proviniera de sus enemigos»; por otra, por el lento avance de la enfermedad que 
no llegó a encender las alertas ni el temor frente al contagio. En este sentido, como informaron los capitanes de amigos en diciembre de 1791, el avance de la viruela era muy lento en esa zona, causando pocos estragos por lo dispersa que se encontraba la población. Al respecto, señalaban que «los indios no le tienen miedo (...) cada día están más distantes de servirse de nuestras medicinas, ni de los religiosos misioneros a quienes miran más como médicos que como pastores espirituales ${ }^{41}$.

INMUNIZAR, ERRADICAR Y UNIVERSALIZAR. LA MIRADA DE LUIS MUÑOZ DE GUZMÁN SOBRE LA INOCULACIÓN A INICIOS DEL SIGLO XIX

Esta forma de entender la inoculación dio un giro importante a inicios del siglo XIX, momento en que el gobernador Luis Muñoz de Guzmán destacó la importancia de difundir la inoculación. En 1803, siete años después de la difusión de la vacuna en Europa y dos años antes de que ésta llegara a Chile, Muñoz de Guzmán envió un reglamento al intendente de Concepción, donde entregó detalles sobre el modo de curar la viruela. Allí dio especial importancia a la inoculación y, por primera vez, lo hizo con un claro énfasis en que más que servir para atacar la epidemia su objeto era prevenir que ésta se desarrollase. Muñoz de Guzmán demostró tener una clara noción respecto a que era posible prevenir el contagio de viruela, de modo que si bien reconoció que «la peste de las viruelas es el azote cruel que despuebla, y desgracia la propagación de sus moradores», "tiene con todo eso la buena cualidad de poder precaver de ante mano casi enteramente el peligro de ella con la inoculación ${ }^{42}$.

En esta instrucción curativa la forma de practicar la inoculación fue distinta a lo que se planteó en la epidemia de 1789, pues Muñoz de Guzmán entendió que la efectividad de la técnica estaba estrechamente vinculada a la masificación de la misma. El primer cambio respecto a cómo se había aplicado anteriormente fue que llamó a que "todos los vecinos y hacendados dispongan que en sus casas y posesiones no haya muchacho de seis años de edad en adelante que no esté inoculado... $»^{43}$. Entender esta práctica en un sentido universal implicó un segundo cambio respecto a las disposiciones anteriores, pues ya no solo los médicos podían administrarla. A su juicio, la inoculación «no es obra que necesita de médicos, pueden y deben ejecutarla las madres con sus hijos». De todos modos, ponía el acento en que el inoculado debía estar en una buena condición de salud, por lo que "las madres y las amas que crían a los niños, deben observar estos y para asegurar mejor le darán cuando vayan a intentar a inoculación un purgatorio suave que los limpie ${ }^{44}$.

La intención de ampliar la cobertura de la inoculación estuvo acompañada de un tercer cambio vinculado a una simplificación del procedimiento, pues el objeto era hacerlo asequible a todos quienes quisieran ponerlo en práctica. A diferencia de lo que años antes planteó el Protomedicato, Muñoz de Guzmán, ya no creyó indispensables los hospitales provisionales de inoculación, sino que enfatizó que el éxito de la operación estaba en la buena conducta del inoculado, en la medida que se procurase «que se mantenga fresco con el vientre corriente a fin de que la calentura no exceda de un grado moderado y que no sea demasiado abundante la erupción ${ }^{45}$. De esta manera, al igual que en los casos anteriores, destacó la importancia "que la viruela que ha de tomar para su operación debe ser de la discreta y de mejor calidad $\aleph^{46}$.

Este cambio tan evidente de la autoridad puede explicarse porque entre fines del siglo XVIII y los primeros años del siglo XIX, se produjo la masificación de la vacuna y con ello se reforzó la idea de que era posible inmunizar al ser humano. Además, se habían desarrollado ideas respecto a que era deber de las autoridades velar por la salud de la población, de modo que siguiendo las directrices de la Corona, se puso de manifiesto una actitud activa frente a la viruela, considerando la variolización un remedio efectivo para prevenirla. De este modo, Muñoz de Guzmán esperaba que la instrucción para curar la viruela que estaba publicando «se propague, la tengan si posible fuere todos los vecinos, la usen en todas partes, se propague por todos los gobiernos, y ranchos... $\Perp^{47}$.

Los marcos de referencia utilizados también habían cambiado, pues si bien frente a la epidemia de 1789 fue el tratado de Francisco Gil el que sirvió de modelo para los tratamientos contra la viruela y la puesta en práctica de la inoculación, en el caso de Luis Muñoz de Guzmán lo fue el Tratado de Medicina Doméstica del doctor Guillermo Buchan ${ }^{48}$. En dicho tratado apareció con claridad la idea de la prevención y la eliminación definitiva de la viruela, lo cual fue un cambio importante, porque ya no solo se trató de curar la viruela o frenar una epidemia, sino de erradicar definitivamente la enfermedad. La variolización era así un tratamiento preventivo en el sentido de que las personas debían inocularse antes de que comenzara a desarrollarse una epidemia, pues solo así sería posible eliminar dicha enfermedad. 


\section{CONCLUSIONES}

Si bien la práctica de inocular se ejercía desde larga data, durante la segunda mitad del siglo XVIII registró un impulso que estuvo dado por su aceptación y difusión en el mundo occidental. El proceso de medicalización que vivían dichas sociedades se tradujo en que la inoculación se introdujo como un tratamiento médico, siendo practicaba por médicos o personas designadas que habían aprendido la técnica. Los documentos que aluden a la inoculación, nos permiten tener certeza de su implementación en la Capitanía General de Chile así como de las discusiones que se suscitaron en torno a ella. Del mismo modo, los oficios sobre la peste de viruela que afectó a Concepción y sus alrededores entre 1789 y 1791, muestran que en Chile también hubo controversias por su aplicación y que al igual que en otros territorios de la América Hispana se debió a la posibilidad de que el inoculado propagara el contagio o bien muriera producto de la variolización.

Para el caso de los territorios hispanoamericanos, es difícil precisar el impacto real de la inoculación, pues tanto en la Capitanía General de Chile, como en Nueva Granada, Nueva España y el Virreinato del Perú lo que vemos son aplicaciones esporádicas vinculadas a la amenaza de epidemia, de modo que la inoculación no fue un remedio preventivo en el sentido que se usara para evitar una epidemia, pues si bien inocular prevenía el contagio, en la práctica se usó como un ele-

\section{NOTAS}

1. Este artículo está basado en un capítulo de mi tesis doctoral titulada "Viruela y Vacuna. Difusión y circulación de una práctica médica. Chile en el contexto hispanoamericano 1780 -1830". Dirigida por el Dr. Rafael Sagredo Baeza y el Dr. Matthew Brown.

2. Autores como Emma Spary, han descrito al siglo XVIII como un periodo de aumento en la medicalización de las sociedades occidentales, lo cual se explica tanto por el mayor poder de los médicos sobre la población, como por un incremento en el compromiso de ésta con la medicina. Michel Foucault, ha definido el proceso de medicalización como "el desarrollo del sistema médico y el modelo seguido por el 'despegue' médico y sanitario de Occidente a partir del siglo XVIII' (Foucault, 1977).

3. Francisco Antonio Cosme Bueno y Alegre, nació en España y hacia 1730 fue enviado al Virreinato del Perú, donde estudió Farmacia y Medicina en la Universidad de San Marcos, graduándose de doctor en 1750 .

4. Véase (Martín y Thomas, 2012). mento más de las medicinas curativas disponibles en aquel momento. En el caso de la Capitanía General de Chile, hemos podido seguir el rastro de la inoculación y de ese modo corroborar que no hubo una organización central que llamara explícitamente a la población a inocularse ni se diseñó una estructura médica que permitiera ponerla en práctica, por lo que no podemos asumir que haya sido una práctica permanente, pero si usada en más de alguna oportunidad. En este sentido, creemos que la relativa aceptación se debió a que la experiencia había demostrado que la técnica era efectiva y los riesgos muy bajos, lo que permitió incorporar la variolización como uno más de los tratamientos destinados a combatir la viruela.

La Instrucción difundida por el gobernador Luís Muñoz de Guzmán representó un cambio de mirada importante, en la medida que manifestó claramente la necesidad de universalizar la inoculación con el fin de aumentar el número de inmunizados. Los bajos riesgos que la experiencia mostró que tenía la variolización, fueron fundamentales en que se promoviera la aplicación de la técnica por quienes quisieran hacerlo. Este tipo de discusiones muestran que a raíz de la variolización se comenzaron a discutir conceptos como prevención y universalidad, enfrentando a las sociedades al tema de la inmunización, nociones centrales en el desarrollo de las medidas posteriores usadas para enfrentar la viruela.

5. A fines del siglo XVIII convivieron dos formas de combatir la viruela, la terapia cálida y la terapia fría. La primera de ellas consistía en someter al enfermo a procedimientos como transpiración, restricción en la circulación del aire en la pieza en que se encontraba, y la casi total privación de líquido. La segunda, proponía permitir al enfermo hidratarse y alimentarse de modo de no perder la vitalidad. También fueron recomendadas las medidas de aislamiento, sangrías y fumigaciones para purificar el aire.

6. Uno de los primeros en referirse a la inoculación fue el padre Feijoo quien transcribió los ensayos ingleses sobre la medida. A él siguieron diversos escritos entre los que destacan (O'Scanlan, 1784) (Joseph Amar, 1774). Para profundizar sobre el tema se sugiere revisar el completo estudio realizado por (León y Dolores Barettino, 2007)

7. Fray Manuel Chaparro y Fray Domingo de Soria trabajaron juntos en Valdivia, pudiendo allí haber intercambiado opiniones y conocimientos respecto a la inoculación.

8. Archivo Nacional Fondo Capitanía General (ANCG), vol. 971, s/f, enero de 1776 
9. Archivo Nacional Fondo Real Audiencia (ANRA), vol. 319, s/f. De acuerdo a los estudios demográficos de Marcelo Carmagnani, en la diócesis de Santiago habitaban hacia 1755 un total de 155.000 personas, lo que equivaldría que el total de inoculados alcanzó al 6,4\%. (Carmagnani, 1967, p.185).

10. Actas de Cabildo de Santiago, 9 de octubre de 1789, disponible en http://historia.uchile.cl/CDA/fh_article/0,1389,SCID\%2 53D18139\%2526ISID\%253D647\%2526JNID\%253D27,00.html

11. ANCG, vol. 811, fj. 29, octubre de 1789.

12. Archivo General de Indias (AGI), Chile, 196, fj. 591, septiembre de 1789. Sobre Juan José Morales, véase (Laval, 1958).

13. AGI, Chile, 196, fj. 591, septiembre de 1789.

14. AGI, Chile, 196, fj. 592, septiembre de 1789.

15. ANCG, vol. 967, fj. 54 y ss.

16. ANCG, vol. 967 , fj. 54 y ss.

17. Actas de Cabildo de Santiago, 9 de octubre de 1789.

18. El fiscal de la Real Audiencia era un funcionario de la Monarquía que podía comunicarse directamente con la corona, sobretodo en cuestiones vinculadas a la tesorería.

19. AGI, Chile, 196, fj. 612, octubre de 1789.

20. AGI, Chile, 196, fj. 612, octubre de 1789 .

21. AGI, Chile, 196, fj. 612, octubre de 1789.

22. AGI, Chile, 196, fj. 612, octubre de 1789.

23. AGI, Chile, 196, fj. 607, octubre de 1789.

24. ANCG, vol. 967, fj. 113, junio de 1790.

25. ANCG, vol. 967, fj. 105- 108, mayo de 1790.

26. AGI, Chile, 196, fj. 604, octubre de 1789.

27. AGI, Chile, 196, fj. 604, octubre de 1789.

28. AGI, Chile, 196, fj. 586, septiembre de 1789.

29. AGI, Chile, 196, fj. 608, octubre de 1789.

30. AGI, Chile, 196, fj. 615, noviembre de 1789.
31. ANCG, vol. 967, fj. 105- 108, mayo de 1790.

32. ANCG, vol. 967 , fj. 108, mayo 1790.

33. ANCG, vol. 967, fj. 108, mayo de 1790.

34. Respecto a si había o no un tiempo más adecuado para inocular los tratadistas del periodo difieren. Por ejemplo, Timoteo O'scanlan señala que se puede inocular en cualquier momento. O'Scalan, planteó que "la inoculación indistintamente tiene prósperas resultas en los climas fríos del norte, como en los ardientes calores del mediodía". (O'Scalan, 1784, p.81).

35. ANCG, vol. 967, fj. 111, junio de 1790.

36. ANCG, vol. 967, fj. 120, julio de 1790.

37. ANCG, vol. 967 , fjs. 120-122, julio de 1790. Participaron Pedro Joseph Salcedo, el Capitán de Dragones Joseph Ruiz, el Ayudante don Gregorio escamilla, los tenientes de Justicia don Peregrino Hortega, Salvador Pradena y Francisco Alarcón, el teniente don Fermín Villagrán y el subdelegado Pedro Lagos, capitán de milicias Joseph Mieres.

38. ANCG, vol. 967, fj. 121, julio de 1791.

39. ANCG, vol. 967, fj. 122, julio de 1791.

40. ANCG, vol. 967, fj. 15, agosto de 1791.

41. ANCG, vol. 967, fj. 29, diciembre de 1791.

42. Luis Muñoz de Guzmán, "Reglamento sobre tratamiento de la viruela", Santiago, 6 de agosto de 1803, Archivo Nacional de Chile, Fondos Varios (en adelante ANFV), vol. 914, fjs.153-155.

43. ANFV, vol. 914, fjs. 153-155.

44. ANFV, vol. 914, fjs. 153-155.

45. ANFV, vol. 914, fjs. 153-155.

46. ANFV, vol. 914, fjs. 153-155.

47. ANFV, vol. 914, fjs. 153-155.

48. La obra de Buchan fue traducida al español por el sacerdote Pedro Sinnot y publicada en Madrid en siete ocasiones entre 1785 y 1798 (Perdiguero, 1989) 


\section{BIBLIOGRAFÍA}

Amar, Joseph (1774) Instrucción curativa de las viruelas, Madrid, Impreso por don Joachim Ibarra.

Amodio, Emanuele (1997), “Curanderos y Médicos Ilustrados. La creación del Protomedicato en Venezuela a fines del siglo XVIII", Asclepio, 49 (1), pp. 95-129.

Barros Arana, Diego (2002), Historia General de Chile, Tomo VII, Santiago, Editorial Universitaria - Centro de Investigaciones Diego Barros Arana.

Bueno, Cosme (1777), Inoculación de las viruela, Lima, Imprenta de los niños huérfanos.

Carmagnani, Marcelo (1967), “Colonial Latin American Demography: Grown of Chilean Population, 1700-1830", Journal of Social History, 1 (2), pp. 179-191.

Carvallo y Goyeneche, Vicente (1875), “Descripción históricogeográfica del Reino de Chile". En Colección de historiadores de Chile y de documentos relativos a la historia nacional, Tomo VIII, Santiago, Imprenta de la librería de El Mercurio.

Casanueva, Fernando (1992), "Una peste de viruelas en la región de la frontera de Guerra hispano-indígena en el Reino de Chile (1791)", Revista de Historia 26 (44), pp. 31-65.

De Demerson, Paula (1993), “La práctica de la variolización en España”, Asclepio, 45 (2), pp. 3-39.

De La Condamine, Charles-Marie (1783), Histoire de l'inoculation de la petite verole, Amsterdam, Societe Typographique.

De Salas, Manuel (1910), Escritos de Don Manuel de Salas: y documentos relativos a él y a su familia, Santiago, Imprenta Cervantes.

Foucault, Michel (1977), "Historia de la Medicalización" Educación Médica y Salud, 11 (1), pp. 3-25.

Gil, Francisco (1784), Disertación Físico Médica en la cual se prescribe un método seguro para preservar a los pueblos de viruelas hasta lograr la completa extinción de ellas en todo el reino, Madrid, Impreso por don Joachim Ibarra.
Gutiérrez, Claudio y Gutiérrez, Flavio (2008), Forjadores de la Ciencia en Chile. Problemas y soluciones, Santiago, Ril.

Laval, Enrique (1958), Noticias Sobre los Médicos en Chile en los Siglo XVI, XVII y XVIII, Santiago, Universidad de Chile.

León, Pilar y Barettino, Dolores (2007), Vicente Ferrer Gorraiz Beaumont y Montesa (1718-1792), un polemista navarro de la Ilustración, Pamplona, Departamento de Salud, Colección Temas de Historia de la Medicina 6.

Martín, Guillermo y Thomas, Hernán (2012), "Inoculaciones y procesiones y cuarentenas. Configuraciones sociotécnicas de las viruelas en América Latina: Funcionamiento y circulación de saberes entre Europa, África y América", Redes 18, pp. 113-142.

O'Scanlan, Timoteo (1774), Práctica moderna de la inoculación con varias observaciones y reflexiones fundadas en ella, precedidas de un discurso sobre la utilidad de esta operación, y un compendio histórico de su origen y de su estado actual, particularmente en España, con un catálogo de algunos inoculados, Madrid, Hilario Santos.

Perdiguero Gil, Enrique (1989), Los tratados de medicina doméstica en la España de la Ilustración, Alicante, Universidad de Alicante.

Piedrola, Gonzalo (1977), “La viruela, primera enfermedad pestilencial prácticamente erradicada en el mundo. Un triunfo de la O.M.S", Anales de la Real Academia Nacional de Medicina, 94 (3), pp. 354-355.

Skold, Peter (1996), "From Inoculation to Vaccination: Smallpox in Sweden in the Eighteenth and Nineteenth Centuries", Population Studies, A Journal of Demography, 50 (2), pp. 247-262.

Spary, Emma (2011), "Health and medicine in the Enlightenment", Mark Jackson (ed.), The Oxford Handbook of The History of Medicine, Oxford, Oxford University Press.

Watts, Sheldon (1997), Epidemics and History. Disease, Power and Imperialism, Wiltshire, Yale University Press. 